\title{
Optimizing glycemic control and minimizing the risk of hypoglycemia in patients with type 2 diabetes
}

\author{
Stanley S Schwartz
}

Affiliate, Main Line Health System, Bryn Mawr, PA; Emeritus, Clinical Associate Professor of Medicine, University of Pennsylvania, Philadelphia, PA

\begin{abstract}
Diabetic microvascular and macrovascular complications arise from hyperglycemia, presenting an increasing healthcare burden as the diabetic population continues to grow. Clinical trial evidence indicates that antihyperglycemic medications are beneficial with regard to microvascular disease (retinopathy, renal impairment, and perhaps neuropathy); however, the benefit of aggressive use of these medications with regard to cardiovascular risk has been less clear in recent studies. These studies were confounded by the propensity of the antihyperglycemic medications involved to cause hypoglycemia, which itself presents cardiovascular risk. This article presents additional context for these seemingly discordant results and maintains that the achievement of glycemic targets is warranted in most patients and provides cardiovascular benefit, provided that hypoglycemia is avoided and the treatment regimen is tailored to the needs of the individual patient. A treatment approach that is driven by these principles and emphasizes diet and exercise, a combination of noninsulin antidiabetic agents, not including sulfonylureas and glinides, and judicious use of insulin is also presented.
\end{abstract}

\section{Citation}

Provenance

Dates

Copyright

Correspondence address

Email address

Competing interests

Abbreviations
Schwartz SS. Optimizing glycemic control and minimizing the risk of hypoglycemia in patients with type 2 diabetes. Drugs in Context 2013; 212255. doi: $10.7573 /$ dic. 212255

Submitted; externally peer reviewed

Submitted: 7 March 2013; Accepted, subject to peer review: 11 March 2013; Published: 22 May 2013

(C) 2013 Schwartz SS. This is an open-access article distributed under the terms of the Creative Commons Attribution License (CC-BY-NC-ND 3.0) which allows unrestricted sharing, copying and distribution for personal use provided it is properly attributed. Commercial use is not permitted.

Stanley S Schwartz MD, 233 E. Lancaster Ave, Suite 233, Ardmore, PA 19003, USA

stschwar@gmail.com

The author has declared the following competing interests: served on advisory boards for Sanofi-Aventis, Takeda, Santarus, Janssen, Amylin, Merck, Novo Nordisk, and Eli Lilly and Company and received honoraria from Bristol-Myers Squibb, AstraZeneca, Takeda, Santarus, Inc., Eli Lilly and Company, Merck, Boehringer Ingelheim, Amylin Pharmaceuticals, and Novo Nordisk.

AACE, American Association of Clinical Endocrinologists; ACCORD, Action to Control Cardiovascular Risk in Diabetes study; ACE, American College of Endocrinology; ADA, American Diabetes Association; ADVANCE, Action in Diabetes and Vascular Disease: Preterax and Diamicron Modified Release Controlled Evaluation study; CHF, congestive heart failure; Cl, confidence interval; CV, cardiovascular; DPP, dipeptidyl peptidase; EASD, European Association for the Study of Diabetes; ECG, electrocardiography; FPG, fasting plasma glucose; GFR, glomerular filtration rate; GLP, glucagon-like peptide; $\mathrm{HbA}$, glycated hemoglobin; $\mathrm{HR}$, hazard ratio; $\mathrm{Ml}$, myocardial infarction; NCS, no-concentratedsweets; NPH, neutral protamine Hagedorn; OR, odds ratio; PPG, postprandial glucose; PROACTIVE, Prospective Pioglitazone Clinical Trial in Macrovascular Events; RR, relative risk; SU, sulfonylurea; T2DM, type 2 diabetes mellitus; UKPDS, United Kingdom Prospective Diabetes Study; VADT, Veterans Affairs Diabetes Trial.

Keywords

clinical management, cardiovascular risk, hypoglycemia, type 2 diabetes mellitus

\section{Introduction}

Diabetes (diagnosed and undiagnosed) affects approximately 25.6 million adults in the US; $90-95 \%$ of these individuals have type 2 diabetes mellitus (T2DM) [1]. An additional 79 million US adults have prediabetes, defined by fasting plasma glucose (FPG) $100-125 \mathrm{mg} / \mathrm{dL}$ or glycated hemoglobin $\left(\mathrm{HbA}_{1 \mathrm{c}}\right)$ levels of $5.7-6.4 \%$ [1]. Compounding the problem, the number of individuals with diagnosed diabetes has increased steadily since the 1990 s, and it is projected to double by 2050 [2].

Because hyperglycemia is associated with the development of micro- and macrovascular complications, achieving glycemic control is crucial to reduce the risk for diabetic complications.

Drugs in Context - The journal of interventions in clinical practice This full text PDF downloaded from www.drugsincontext.com Drugs in Context 2013; 212255. ISSN 1740-4398

Copyright (C) 2013 Schwartz SS. Distributed under the terms of the Creative Commons Attribution License CC-BY-NC-ND 3.0.

No other uses without permission.
However, the potential for medication-induced hypoglycemia and its negative sequelae present a barrier to achieving glycemic targets. Insulin and the sulfonylureas (SUs) are the main cause of hypoglycemia in patients with T2DM [3]. An estimated $9.7 \%$ of overall adverse drug events treated in emergency departments over the 2-year period 2004-2005 were attributable to insulin and oral antihyperglycemic agents [4]. Moreover, in the elderly, these drugs accounted for $24.6 \%$ of such events in the 3-year period 2007-2009, of which $94.6 \%$ were ascribed to hypoglycemia [5].

The purpose of this article is to discuss recent data regarding the optimal degree of glycemic control in light of the possibility of medication-induced hypoglycemia, and to discuss appropriate goal setting that considers patient-specific factors. Treatment recommendations that address the balance among glycemic control, the risk of hypoglycemia, and the individual needs of the patient are discussed. 


\section{Clinical consequences of treatment- induced hypoglycemia}

\section{Symptoms}

A decline in blood glucose (to $65-70 \mathrm{mg} / \mathrm{dL}$ ) leads to suppression of insulin production, accompanied by a rise in the counterregulatory hormones glucagon, growth hormone, steroids, and epinephrine [6,7]. Symptoms of hypoglycemia are initiated by epinephrine release and may begin when blood glucose falls $[6,7]$. Neuroglycopenic symptoms include faintness, dizziness, blurred vision, impaired concentration, and tingling, whereas autonomic symptoms include anxiety, palpitation, hunger, sweating, irritability, and tremor [8].

\section{Cardiovascular effects}

Acute cardiovascular (CV) effects of hypoglycemia are secondary to epinephrine release and include increased blood flow, heart rate, systolic blood pressure, stroke volume, and cardiac output. Hypoglycemic episodes have been associated with prolonged electrocardiogram QT intervals, rhythm disturbances, and cardiac ischemia; these mechanisms link hypoglycemia with poor CV outcomes $[9,10]$. Hypoglycemia has been documented at the time of sudden death in type 1 diabetes and has been linked to ECG and rhythm abnormalities [10,11].

\section{Neurologic effects}

The neurologic effects of hypoglycemia include altered hypoglycemic responses and dementia. Recurrent hypoglycemia affects counter-regulatory mechanisms such that a larger reduction in blood glucose is required to trigger hormonal counter-regulation and onset of symptoms [6]. This response results in increased hypoglycemia unawareness, promoting further hypoglycemic episodes. The relationship between severe hypoglycemic episodes (resulting in an emergency department visit or hospitalization) and dementia in an older (mean age 65 years) cohort of T2DM patients was examined in a diabetes registry study [12]. Risk of dementia increased as the number of hypoglycemic episodes increased from one episode (HR: 1.26, 95\% CI: 1.1-1.49) to $\geq 3$ episodes (HR: 1.94, 95\% CI: 1.42-2.64) during the 22-year observation period.

\section{Risk factors for hypoglycemia}

Age is an important risk factor for hypoglycemia, as biochemical studies have documented impaired counter-regulatory epinephrine and glucagon responses to hypoglycemia in elderly patients $[13,14]$. Hypoglycemia unawareness is also more common in the elderly; in a study involving experimentally induced hypoglycemia, elderly T2DM patients reported lower autonomic and neuroglycopenic symptom scores than did middle-aged T2DM patients [8].

Other risk factors for hypoglycemia include missing meals, coronary artery disease, renal impairment, insulin or SU treatment, and a history of severe hypoglycemia. A retrospective chart review of 244 T2DM patients (mean age 74 years) admitted to the hospital for hypoglycemia demonstrated that - after controlling for age and gender - significant risk factors for hypo- glycemia were skipping a recent meal (odds ratio [OR]: 3.93 , 95\% CI: $1.82-8.50 ; p<0.001)$ and coronary artery disease (OR: 2.3, 95\% CI: 1.04-5.10; $p=0.041$ ) [15]. An Australian study of 616 patients with diabetes (mean age 67 years) indicated that determinants of severe hypoglycemic episodes included diabetes duration $\geq 8$ years (HR: $2.92,95 \%$ CI: $1.6-5.32$; $p<0.001$ ), treatment with an SU (HR: 2.50, 95\% CI: 1.16-5.38; $p=0.019$ ) or insulin (HR: 4.29, 95\% CI: 2.44-7.55; $p<0.001$ ), duration of insulin treatment (HR: $1.42,95 \%$ CI: $1.24-1.63$; $p<0.001$ ), renal impairment (HR: 2.39, 95\% CI: 1.37-4.15; $p=0.002$ ), and history of severe hypoglycemia (HR: 5.66, 95\% CI: 2.21-14.50; $p<0.001)$ [16]. Exercise increases the risk of hypoglycemia because it increases glucose utilization [6]. Additional risk factors include higher $\mathrm{HbA}_{1 \mathrm{c}}$, lower FPG, and psychiatric problems, particularly dementia and cognitive impairment [17].

\section{Importance of optimizing glycemic control}

It is well established that hyperglycemia increases the risk of microvascular (e.g., nephropathy, retinopathy, neuropathy) and macrovascular (e.g., stroke, myocardial infarction [MI], CV death) complications in patients with T2DM. The United Kingdom Prospective Diabetes Study (UKPDS) documented that the rates of diabetes-related complications - including mortality, stroke, MI, amputation, and heart failure - steadily increase as $\mathrm{HbA}_{1 \mathrm{c}}$ level increases from $<6 \%$ to $\geq 10 \%$ [18]. These complications are thought to be caused by metabolic pathways downstream from intracellular hyperglycemia that include oxidative stress, endothelial dysfunction, and inflammation, among other mechanisms [19].

There is also evidence that macrovascular complications such as coronary heart disease, MI, and stroke - may occur in patients who are hyperglycemic but have not yet reached the glycemic threshold for diagnosis of T2DM (American Diabetes Association [ADA] diagnostic criteria for T2DM, FPG $\geq 126 \mathrm{mg} / \mathrm{dL}$ or $\mathrm{HbA}_{1 \mathrm{c}} \geq 6.5 \%$; definition of prediabetes, $\mathrm{HbA}_{1 \mathrm{c}}$ $5.7-6.4 \%$ [20]). For example, a meta-regression analysis of 20 studies in more than 95,000 subjects found a relationship between blood glucose levels and the occurrence of CV events when analyzed over a 12-year follow-up period, even in nondiabetic subjects (for subjects with impaired glucose tolerance [2-hour postprandial glucose [PPG] threshold level $140 \mathrm{mg} /$ $\mathrm{dL}$ ], relative risk [RR]: $1.58,95 \%$ CI: 1.19-2.10) [21]. Consistent with this finding, a study of a Japanese population-based cohort $>40$ years of age $(\mathrm{N}=2534)$ found that patients with impaired glucose tolerance had a higher risk of CV death compared with those with normal glucose tolerance (OR: 2.303, 95\% CI: 1.022-5.188) [22]. Taken together, these studies support the idea that hyperglycemia is a continuous risk factor for CV mortality: risk gradually increases as the duration and level of hyperglycemia increase, rather than being associated with a specific glycemic level above the diabetic threshold. These observations support the rationale for early treatment of hyperglycemia.

An early study confirmed the prediction that lowering $\mathrm{HbA}_{1 \mathrm{c}}$ levels with pharmacotherapy would positively impact vascular 
outcomes. The UKPDS initially found a reduced risk for vascular complications among patients treated with an SU or insulin compared with dietary intervention alone; pharmacotherapy reduced microvascular risk by $25 \%(p=0.0099)$ and macrovascular risk by $16 \%(p=0.052)$ [23]. A subsequent follow-up study of the UKPDS found that the reduction in risk for vascular complications among patients receiving pharmacotherapy compared with dietary intervention was maintained after 10 years [24]. For patients who had received SU or insulin, there was a significant reduction in risk for any diabetes-related outcome (RR: 0.91, 95\% CI: $0.83-0.99 ; p=0.04$ ), and in microvascular risk (RR: $0.76,95 \%$ CI: $0.64-0.89$; $p=0.001)$ at 10 years. These results emphasize an important role for establishing glycemic control in reducing the long-term risk of vascular sequelae [24].

In contrast, several recent, large trials have provided conflicting evidence in T2DM patients [25-27]. The use of intensive therapy to achieve lower glycemic targets in these studies failed to show a demonstrable benefit with regard to macrovascular complications; however, interpretation of these results was complicated by adverse effects (e.g., hypoglycemia, weight gain) associated with the particular therapies used in the intensive treatment groups (i.e., SUs and insulin). Results of these studies are summarized in the following section.

The Action to Control Cardiovascular Risk in Diabetes (ACCORD) study evaluated 10,251 patients with a mean age of 62 years, mean T2DM duration of 10 years, and baseline $\mathrm{HbA}_{1 \mathrm{c}}$ levels of $8.3 \%$; target $\mathrm{HbA}_{1 \mathrm{c}}$ levels for the intensive therapy and standard therapy groups were $<6.0 \%$ and $7.0-7.9 \%$, respectively [25]. More patients in the intensive therapy group than in the standard therapy group were treated with glimepiride (78.2 vs 67.6\%), repaglinide (50.2 vs $17.7 \%$ ), and insulin (77.3 vs $55.4 \%)$. Patients in the intensive therapy group experienced a greater incidence of hypoglycemia (16.2 vs $5.1 \%$; $p<0.001)$ than those in the standard therapy group. Further, patients in the intensive therapy group experienced greater weight gain than those in the standard therapy group $(3.5 \mathrm{~kg} v s 0.4 \mathrm{~kg})$, and a larger proportion of patients in the intensive therapy group gained $>10 \mathrm{~kg}$ (27.8 vs $14.1 \%$, respectively; $p<0.001)$. The risk of death from CV causes was significantly higher for the intensive therapy group compared with the conventional therapy group (2.6 vs 1.8\%; HR: $1.35,95 \%$ CI: $1.04-1.76 ; p=0.02)$, as was the risk of death from any cause ( 5.0 vs $4.0 \%$; HR: 1.22 , 95\% CI: $1.01-1.46 ; p=0.04$ ), leading to early termination of the study [25].

The Action in Diabetes and Vascular Disease: Preterax and Diamicron Modified Release Controlled Evaluation (ADVANCE) study evaluated 11,140 patients with a mean age of 66 years and T2DM duration of 8 years; the glycemic target for the intensive therapy group was $\leq 6.5 \%$, whereas that of the standard therapy group was based on local guidelines. At the end of follow-up, mean $\mathrm{HbA}_{1 \mathrm{c}}$ levels had decreased from a baseline of $7.51 \%$ and $7.52 \%$ to $6.5 \%$ and $7.3 \%$ for the intensive and standard therapy groups, respectively [26]. Compared with the standard therapy group, the intensive therapy group had a reduced risk of microvascular complications (HR: $0.86,95 \%$ CI $0.77-0.97$; $p=0.01$ ) but no change in risk of macrovascular complications (HR: 0.94, 95\% CI: 0.84-1.06; $p=0.32$ ). SUs were used to treat $92.4 \%$ of patients in the intensive therapy group and $58.7 \%$ in the standard therapy group; insulin use was reported by $40.5 \%$ and $24.1 \%$ of patients in the intensive therapy and standard therapy groups, respectively. Compared with the standard therapy group, the intensive therapy group experienced a greater rate of minor hypoglycemia (120 vs 90 events per 100 patient-years) and severe hypoglycemia ( 0.7 vs 0.4 events per 100 patient-years; $p<0.001)$. Moreover, whereas both intensive and standard therapy groups had similar body weights at baseline (78.2 and $78.0 \mathrm{~kg}$, respectively), the intensive therapy group had significantly greater mean body weight versus the standard therapy group during the follow-up period $(0.7 \mathrm{~kg}$ greater; $p<0.001)$ [26]. Factors of weight gain and hypoglycemia may have limited the extent of macrovascular benefits of antihyperglycemic therapy in the intensive therapy group.

Similarly, the Veterans Affairs Diabetes Trial (VADT) of 1791 veterans with diabetes and mean baseline $\mathrm{HbA}_{1 \mathrm{c}}$ of $9.4 \%$ reported greater weight gain $(8.2 \mathrm{~kg} v s 4.1 \mathrm{~kg} ; p=0.01)$ and more hypoglycemic events (1566 vs 432 per 100 patient-years; $p<0.001$ ) in the intensive therapy group compared with the standard therapy group. $\mathrm{HbA}_{1 c}$ targets for these patients were 1.5 percentage points lower than the standard therapy group. This goal was reached; mean $\mathrm{HbA}_{1 \mathrm{c}}$ in each group was $6.9 \%$ and $8.4 \%$, respectively [27]. Compared with the standard therapy group, the rate of $\mathrm{CV}$ events in the intensive therapy group was $11.9 \%$ lower (29.5 vs 33.5\%). However, no significant differences between groups were observed in the time to first $\mathrm{CV}$ event (HR: 0.88, 95\% CI: $0.74-1.05 ; p=0.14)$ or in the time to CV-related death (HR: 1.32 , 95\% CI: 0.81-2.14; $p=0.26$ ) [27].

\section{Relationship between hypoglycemia and negative CV outcomes}

Further examination of data from the ACCORD, ADVANCE, and VADT studies has linked the occurrence of hypoglycemic episodes and persistently high $\mathrm{HbA}_{1 \mathrm{c}}$ with negative $\mathrm{CV}$ outcomes, underscoring the need to optimize glycemic control by lowering $\mathrm{HbA}_{1 \mathrm{c}}$ while minimizing the risk of hypoglycemia. In ACCORD and ADVANCE, episodes of severe hypoglycemia were associated with microvascular events, macrovascular events, and death $[28,29]$. Furthermore, a single episode of severe hypoglycemia may be sufficient to raise the risk of negative outcomes. In ADVANCE, there was no dose-response relationship between the number of severe hypoglycemic episodes and macrovascular events, microvascular events, cardiovascular death, or death from any cause [29]. The authors concluded that, if not causative, severe hypoglycemia could be a marker for vulnerability to negative vascular outcomes [29]. It should also be noted that antihyperglycemic treatment failure was also linked to negative $\mathrm{CV}$ outcomes in the ACCORD study. Post hoc analyses showed that increased mortality occurred primarily in patients who failed to respond to intensive therapy rather than in those who achieved glycemic control with intensive therapy [30]. In sum, these analyses suggest that with intensive therapy, both hypoglycemic episodes and failure to adequately reduce blood sugar, rather than aggressive glycemic control per se, are associated with negative outcomes [30]. These data also raise the point that these two groups of patients - those with treatment failure and those with a propensity 
for severe hypoglycemia - may be higher-risk groups that require special consideration when selecting a treatment regimen.

Recent meta-analyses of these and other trials using various treatment regimens for T2DM have also assessed the impact of improved glycemic control on CV outcomes. Several analyses of five trials (ACCORD, ADVANCE, UKPDS, and VADT, plus the Prospective Pioglitazone Clinical Trial in Macrovascular Events [PROACTIVE], which compared pioglitazone with placebo) revealed that intensive therapy reduced the incidence of $\mathrm{MI}$ (OR: 0.849, 95\% CI: 0.778-0.926), non-fatal MI (OR: 0.83, 95\% CI: $0.75-0.93$ ), and coronary heart disease (OR: 0.85, 95\% CI: 0.77-0.93), but not stroke (OR: 0.93, 95\% CI: 0.81-1.06) or all-cause mortality (OR: 1.02, 95\% CI: 0.87-1.19) [31,32]. There was also a significant correlation between the risk of severe hypoglycemia and that of CV mortality with intensive treatment [31,32]. Another meta-analysis of 13 trials compared intensive oral or insulin therapy with standard care, less intensive treatment, or placebo and found similar results: intensive treatment reduced the risk of non-fatal MI and microalbuminuria, and increased the risk of severe hypoglycemia more than two-fold compared with the less intensive alternatives. There was no effect on CV death or all-cause death [33]. Together, these analyses demonstrate the $\mathrm{CV}$ benefit of tighter glycemic control, while suggesting that avoidance of hypoglycemia is important for fully realizing that benefit.

Although ACCORD, ADVANCE, VADT, and UKPDS all used therapies that have the potential to induce hypoglycemia and weight gain, differences among the study populations may explain why better outcomes were observed with respect to macrovascular risk and intensive therapy in UKPDS versus the other three studies. Compared with ACCORD, ADVANCE, and VADT, UKPDS participants were younger (mean age 53 years vs 62, 66, and 60 years, respectively) and had lower baseline $\mathrm{HbA}_{1 \mathrm{c}}$ (achieved after a dietary run-in period in UKPDS; 7.1 vs $8.3 \%, 7.5 \%$, and 9.4\%) [23,25-27]. Notably, the duration of time from diagnosis of T2DM among patients in the ACCORD (median, 10 years), ADVANCE (approximate mean 8.0 years), and VADT (mean 11.5 years) was considerably longer than those enrolled in the UKPDS, as the UKPDS patients were newly diagnosed [23,2527]. Thus, age, disease duration, and severity may have amplified the negative effects of medication-induced hypoglycemia on macrovascular risk in ACCORD, ADVANCE, and VADT. Consequently, clinicians might consider pursuing glycemic control in elderly patients and those with preexisting CV disease or more advanced T2DM with medications that effectively treat hyperglycemia but strictly avoid hypoglycemia and weight gain [34].

\section{Treatment approach and considerations}

The American Diabetes Association/European Association for the Study of Diabetes (ADA/EASD) position statement and the American Association of Clinical Endocrinologists and American College of Endocrinology (AACE/ACE) guidelines emphasize the importance of individualizing diabetes treatment based on the safety profile and level of glycemic control achieved with various agents [35-37]. Metformin remains the standard first-line agent based on its $\mathrm{HbA}_{1 c}$-lowering efficacy and low incidence of hypoglycemia and weight gain; however, in patients for whom metformin is contraindicated or not tolerated, other agents may be used as first-line therapy, including incretin-based therapies (e.g., glucagon-like peptide [GLP]-1 receptor agonists and dipeptidyl peptidase-4 [DPP-4] inhibitors), pioglitazone, $\alpha$-glucosidase inhibitors, colesevelam, and bromocriptine [36,37]. ADA/EASD also suggest that SUs may be used as firstline therapy in these patients, although the AACE/ACE guidelines do not. In patients advancing to combination therapy, a DPP-4 inhibitor, GLP-1 receptor agonist, pioglitazone, SU, or basal insulin may be added to metformin according to the $\mathrm{ADA} / \mathrm{EASD}$ recommendations; however, the AACE/ACE guidelines do not recommend insulin as a second-line agent and note the risk of hypoglycemia associated with SUs. The AACE/ACE guidelines also suggest combining metformin with colesevelam or an $\alpha$-glucosidase inhibitor [36]. Finally, the AACE/ACE guidelines specify that in patients requiring insulin, analog insulins are preferred, as the time course of the action of regular human insulin and neutral protamine Hagedorn (NPH) insulin does not adequately mimic the normal physiologic time course of insulin, making them more likely to cause hypoglycemia [36].

With respect to avoiding hypoglycemia, The Endocrine Society guidelines recommend consideration of conventional risk factors (insulin dosing, decreased glucose production or delivery, increased glucose utilization or insulin sensitivity, decreased insulin clearance) and factors indicating compromised hypoglycemia response mechanisms (history of severe hypoglycemia, hypoglycemia unawareness, degree of endogenous insulin deficiency) when selecting therapy [38].

\section{Special populations}

Current guidelines emphasize that risks of each medication should be considered together with patient-specific factors [36,37]. Pioglitazone should be avoided in patients with Class 3 or 4 congestive heart failure (CHF) and chronic kidney disease, as it may cause weight gain, fluid retention, and CHF [36,39]. SUs and insulin have a propensity to cause hypoglycemia and weight gain, and should be avoided especially in patients at risk for hypoglycemia (e.g., patients with renal impairment) [37]. For patients with renal disease, metformin should not be used if glomerular filtration rate (GFR) is $<30 \mathrm{~mL} /$ minute, and a reduced dose is advised for GFR $<45 \mathrm{~mL} /$ minute [37]. Further, the National Kidney Foundation recommends that exenatide not be used in patients with GFR $<30 \mathrm{~mL} /$ minute and liraglutide not be used in patients with GFR $<60 \mathrm{~mL} /$ minute. Most DPP-4 inhibitors require dose adjustment for patients with renal impairment [39].

\section{Author's perspective on treatment approach}

The following approach to T2DM treatment has been gleaned from the author's experience (Table 1 and Figure 1). This approach stresses the importance of diet and exercise, as well as the judicious use of antidiabetic medications to reduce $\mathrm{HbA}_{1 \mathrm{c}}$ while avoiding hypoglycemia and weight gain. It is emphasized that this perspective is the author's personal opinion based on his clinical experience and should be balanced against the recommendations of current clinical guidelines [35-37]. 
Table 1. Author's general principles of treatment for type 2 diabetes*

- Target $\mathrm{HbA}_{1 \mathrm{c}} \leq 6.5 \%{ }^{+}$

- Lifestyle modification is essential; no smoking

- Minimize risk/severity of hypoglycemia and weight gain

- Address FPG and PPG

- Fast therapeutic change (1-2 months)

- Combination therapy frequently required; choose drugs with complementary mechanisms of action

- When using insulin, add insulin-sensitizing agent(s)

- Safety and efficacy most important; cost addressed on an individual basis

- Therapeutic choice should match drug characteristics with patient characteristics

*Based on author's clinical experience; not evaluated in a clinical trial. ${ }^{+} \mathrm{HbA}_{1 \mathrm{c}}$ goals should be set as close to $6.5 \%$ as possible while still considering comorbidities and clinical characteristics.

Abbreviations

FPG, fasting plasma glucose; $\mathrm{HbA}_{1 c^{\prime}}$ glycated hemoglobin $\mathrm{A}_{1 c^{\prime}}$ PPG, postprandial glucose.

doi: 10.7573/dic.212255.t001

The choice of therapy, as well as the speed and extent to which glycemic control is achieved, must be tailored to the individual patient, with special consideration given to those with renal impairment or who are elderly, obese, or have a longer history of T2DM. In addition to guidance on special populations put forth by the ADA/EASD and ACE/AACE, the author adds that pioglitazone should be used carefully and in conjunction with a low-salt diet in patients with peripheral edema, and avoided in patients with a history of bladder cancer. Intensive glycemic control (with patient-specific modifications) is warranted as long as drugs that have the potential to induce hypoglycemia (i.e., SUs and insulin) are avoided or are carefully monitored if they are to be part of the therapeutic regimen. Management goals in T2DM should also address issues of overweight/obesity and other cardiovascular risk factors to improve long-term outcomes.

The author's preferred approach is to try several therapies that have a low potential to cause hypoglycemia before resorting to insulin use. Seven classes of antihyperglycemic agents are associated with a neutral or low risk of hypoglycemia (biguanides [metformin], DPP-4 inhibitors, GLP-1 receptor agonists, thiazolidinediones, $\alpha$-glucosidase inhibitors, bile acid sequestrants, and dopamine-2 agonists), primarily because they do not act directly to increase insulin levels in a glucose-independent manner. Although not indicated for T2DM, the anti-anginal ranolazine has been shown to further decrease $\mathrm{HbA}_{1 \mathrm{c}}$ when added to current T2DM treatment, also in a glucose-dependent manner [40].

In most situations, avoiding hypoglycemia is accomplished empirically, based on the clinician's experience, with careful attention to comorbid conditions that can modify these risk factors (e.g., liver and renal disease, exercise, other medications). Questioning and evaluation of the patient for indications of possible hypoglycemic unawareness (e.g., a pattern of high FPG for several days and normal FPG for several days; morning wakening with a headache, nightmares, vivid dreams, or sweats, even after

Figure 1. Author's approach to T2DM treatment.

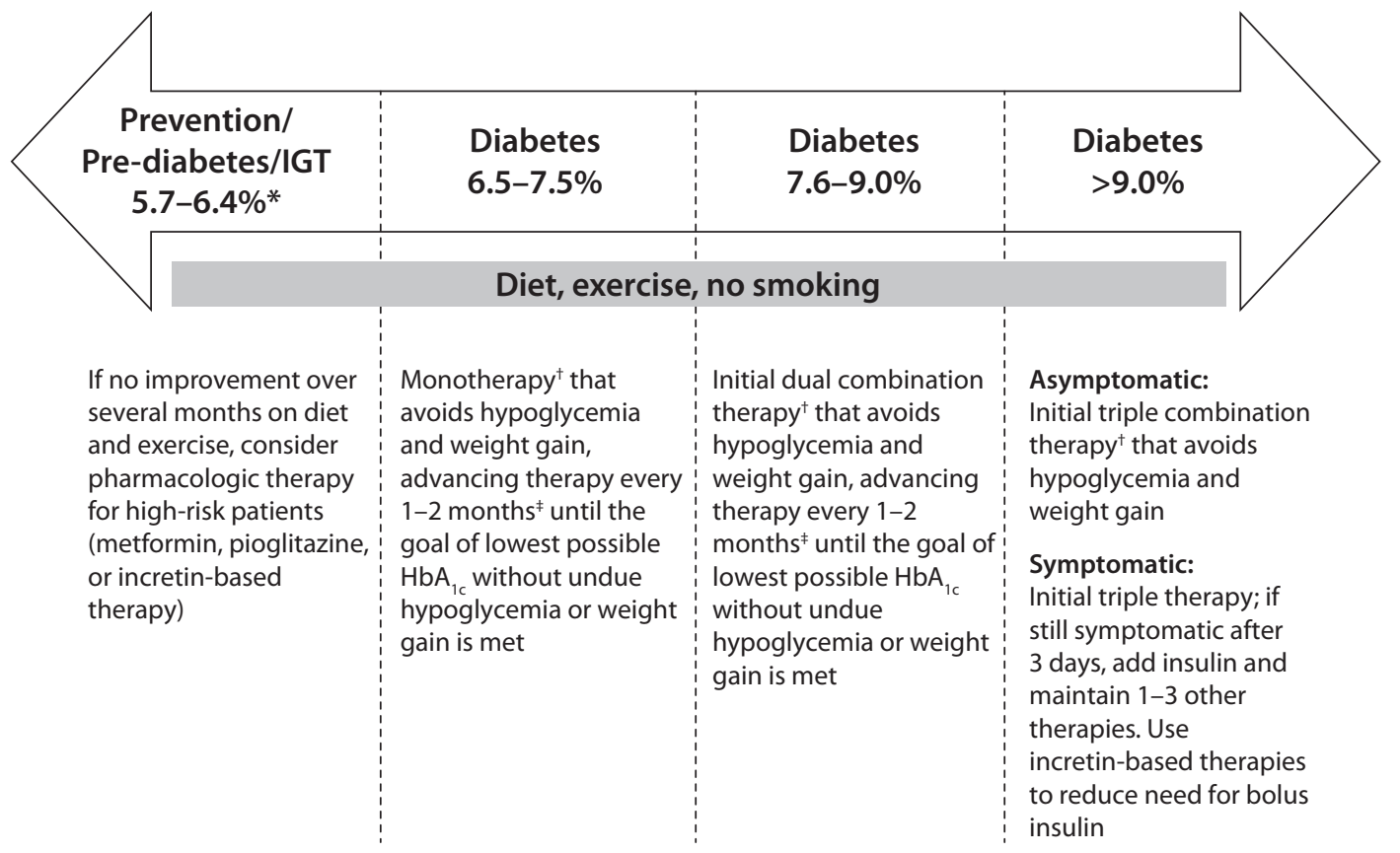

${ }^{*} \mathrm{HbA}_{1 \mathrm{c}}$

${ }^{\dagger}$ Potential agents: metformin, DPP-4 inhibitor, GLP-1 receptor agonist, colsevelam, acarbose, ranolazine, bromocriptine, and sodium-glucose cotransporter- 2 inhibitors could be considered.

‡Early therapeutic changes (every 1-2 months) can be identified with agreesive monitoring.

Abbreviations

IGT, impaired glucose tolerance

doi: 10.7573/dic.212255.f001 
sleeping through the night; terrible hunger prior to meals requiring increased food intake/extra snacks) is critical.

\section{Author's stepwise approach to adjusting insulin and diet to prevent hypoglycemia}

Regardless of the particular guidelines used, selection of the therapeutic regimen should be tailored to the needs of the individual patient. Insulin should be avoided or delayed in patients not adhering to a minimum no-concentrated-sweets (NCS) diet (Table 2) unless they are symptomatic. Patients with symptomatic diabetes (e.g., polydipsia, polyuria, and polyphagia) should be treated with insulin in conjunction with diet. If insulin is necessary, non-insulin therapies (especially agents that have a PPG-lowering effect) should be maintained to reduce glycemic variability and the need for bolus insulin [36].

In patients who come to a physician already receiving insulin and consuming simple sugars, the author's goal is to try to reduce or discontinue the use of bolus insulin dosing. If the patient is willing to commit to the NCS diet, the insulin dose may be decreased by $25 \%$; if the physician ascertains (through careful questioning) that the patient has likely experienced hypoglycemia, consider decreasing the insulin dose by an additional $25 \%$.

Table 2. Author's no-concentrated-sweets diet*

\begin{tabular}{l}
\hline Allowable carbohydrates (in moderation) ${ }^{\dagger}$ \\
\hline Bread \\
Rice \\
Potatoes \\
Pasta \\
Corn \\
1 whole fresh fruit (apple, pear, banana, hard plum, hard peach) or \\
handful of small fruit (grapes, cherries) taken with meal \\
"Sugar-free" or "no sugar added" foods with $\leq 10$ kcal per serving
\end{tabular}

Not recommended ${ }^{\ddagger}$

Candy

Cookies

Cake

Ice cream

Pies

Sweet sodas

Juices, unless they are "sugar-free" or "no sugar added" with $\leq 10 \mathrm{kcal}$ per serving

"Sugar-free" or "no sugar added" foods with $>60$ kcal per serving ${ }^{\S}$ Watery fruits (watermelon, honeydew, oranges, ripe cantaloupe, ripe peaches, ripe plums, applesauce, fruit cocktails)

Dried fruits

*Based on author's clinical experience; not evaluated in a clinical trial. List is not all-inclusive.

'Induce a slow glycemic rise that can be controlled with available oral therapies.

"Induce a rapid glycemic rise uncontrolled by oral therapies; avoidance of these foods will also help decrease body weight.

sUsually contain other simple sugars (i.e., fructose, sucrose, maltose). doi: 10.7573/dic.212255.t002
Incretin-based therapies can be used to prevent or reduce the need for bolus insulin. Some patients may be titrated off insulin as the clinician adds or switches to therapies that reduce the insulin requirement (metformin, DPP-4 inhibitors, GLP-1 receptor agonists, or pioglitazone) or as the patient achieves weight loss.

For patients on a stable dose of insulin who undertake weight loss efforts, the insulin dose may be decreased proportionally to the reduction in caloric intake. Reductions in insulin dose are especially warranted in patients who began insulin therapy without strict dietary changes or when the newer antihyperglycemic agents were not yet available.

\section{Summary}

It is well established that hyperglycemia is associated with microvascular and macrovascular complications in patients with T2DM. Glycemic control may reduce the risk of macrovascular complications; however, recent studies suggest that this benefit may depend in part on selecting appropriate therapeutic regimens with a lower risk of causing hypoglycemia. Management strategies that employ diet and exercise interventions, early treatment, and combination therapy with metformin, incretin-based therapies (DPP-4 inhibitors and GLP-1 receptor agonists), colesevelam, and/or bromocriptine, as well as judicious use of insulin, may help patients to avoid hypoglycemia and weight gain and therefore maximize the CV benefits of glycemic control.

\section{Acknowledgment and funding declaration}

Medical writing support for the preparation of this manuscript was provided by Valerie P. Zediak, PhD and Erica S. Wehner, RPh, CMPP, from Complete Healthcare Communications, Inc., with funding from Bristol-Myers Squibb and AstraZeneca.

\section{Author Contributions}

S Schwartz made substantial intellectual contributions to manuscript content and development, interpretation of data, and review and final approval of submitted manuscript.

\section{References}

1. Centers for Disease Control and Prevention. National diabetes fact sheet: national estimates and general information on diabetes and prediabetes in the United States, 2011. Atlanta, GA: U.S. Department of Health and Human Services, Centers for Disease Control and Prevention, 2011. Available at: http://www.cdc.gov/diabetes/pubs/pdf/ndfs_2011.pdf. [Last accessed 8 January, 2013].

2. Boyle JP, Thompson TJ, Gregg EW, Barker LE, Williamson DF. Projection of the year 2050 burden of diabetes in the US adult population: dynamic modeling of incidence, mortality, and prediabetes prevalence. Popul Health Metr 2010;8:29.

3. Amiel SA, Dixon T, Mann R, Jameson K. Hypoglycaemia in type 2 diabetes. Diabet Med 2008;25:245-54. 
4. Budnitz DS, Pollock DA, Weidenbach KN, Mendelsohn AB, Schroeder TJ, Annest JL. National surveillance of emergency department visits for outpatient adverse drug events. JAMA 2006;296:1858-66.

5. Budnitz DS, Lovegrove MC, Shehab N, Richards CL. Emergency hospitalizations for adverse drug events in older Americans. N Engl J Med 2011;365:2002-12.

6. Cryer PE, Davis SN, Shamoon H. Hypoglycemia in diabetes. Diabetes Care 2003;26:1902-12.

7. Zammitt NN, Frier BM. Hypoglycemia in type 2 diabetes: pathophysiology, frequency, and effects of different treatment modalities. Diabetes Care 2005;28:2948-61.

8. Bremer JP, Jauch-Chara K, Hallschmid M, Schmid S, Schultes B. Hypoglycemia unawareness in older compared with middle-aged patients with type 2 diabetes. Diabetes Care 2009;32:1513-7.

9. Desouza C, Salazar H, Cheong B, Murgo J, Fonseca V. Association of hypoglycemia and cardiac ischemia: a study based on continuous monitoring. Diabetes Care 2003;26:1485-9.

10. Gill GV, Woodward A, Casson IF, Weston PJ. Cardiac arrhythmia and nocturnal hypoglycaemia in type 1 diabetes-the 'dead in bed' syndrome revisited. Diabetologia 2009;52:42-5.

11. Tanenberg RJ, Newton CA, Drake AJ. Confirmation of hypoglycemia in the "dead-in-bed" syndrome, as captured by a retrospective continuous glucose monitoring system. Endocr Pract 2010;16:244-8.

12. Whitmer RA, Karter AJ, Yaffe K, Quesenberry CP Jr., Selby JV. Hypoglycemic episodes and risk of dementia in older patients with type 2 diabetes mellitus. JAMA 2009;301:156572.

13. Ortiz-Alonso FJ, Galecki A, Herman WH, Smith MJ, Jacquez JA, Halter JB. Hypoglycemia counterregulation in elderly humans: relationship to glucose levels. Am J Physiol 1994;267(4 Pt 1):E497-E506.

14. Marker JC, Clutter WE, Cryer PE. Reduced epinephrine clearance and glycemic sensitivity to epinephrine in older individuals. Am J Physiol 1998;275(5 Pt 1):E770-6.

15. Lin YY, Hsu CW, Sheu WH, Chu SJ, Wu CP, Tsai SH. Risk factors for recurrent hypoglycemia in hospitalized diabetic patients admitted for severe hypoglycemia. Yonsei Med J 2010;51:367-74.

16. Davis TM, Brown SG, Jacobs IG, Bulsara M, Bruce DG, Davis WA. Determinants of severe hypoglycemia complicating type 2 diabetes: the Fremantle Diabetes Study. J Clin Endocrinol Metab 2010;95:2240-7.

17. Feil DG, Rajan M, Soroka O, Tseng CL, Miller DR, Pogach LM. Risk of hypoglycemia in older veterans with dementia and cognitive impairment: implications for practice and policy. J Am Geriatr Soc 2011;59:2263-72.

18. Stratton IM, Adler AI, Neil HA, et al. Association of glycaemia with macrovascular and microvascular complications of type 2 diabetes (UKPDS 35): prospective observational study. BMJ 2000;321(7258):405-12.

19. Brownlee M. The pathobiology of diabetic complications: a unifying mechanism. Diabetes 2005;54:1615-25.

20. American Diabetes Association. Standards of medical care in diabetes-2013. Diabetes Care 2013;36(Suppl 1):S11-S66.
21. Coutinho M, Gerstein HC, Wang Y, Yusuf S. The relationship between glucose and incident cardiovascular events. A metaregression analysis of published data from 20 studies of 95,783 individuals followed for 12.4 years. Diabetes Care 1999;22:233-40.

22. Tominaga M, Eguchi H, Manaka H, Igarashi K, Kato T, Sekikawa A. Impaired glucose tolerance is a risk factor for cardiovascular disease, but not impaired fasting glucose. The Funagata Diabetes Study. Diabetes Care 1999;22:920-4.

23. UK Prospective Diabetes Study (UKPDS) Group. Intensive blood-glucose control with sulphonylureas or insulin compared with conventional treatment and risk of complications in patients with type 2 diabetes (UKPDS 33). Lancet 1998;352(9131):837-53.

24. Holman RR, Paul SK, Bethel MA, Matthews DR, Neil HA. 10-year follow-up of intensive glucose control in type 2 diabetes. N Engl J Med 2008;359:1577-89.

25. Gerstein HC, Miller ME, Byington RP, et al. Effects of intensive glucose lowering in type 2 diabetes. $\mathrm{N}$ Engl J Med 2008;358:2545-59.

26. Patel A, MacMahon S, Chalmers J, et al. Intensive blood glucose control and vascular outcomes in patients with type 2 diabetes. N Engl J Med 2008;358:2560-72.

27. Duckworth W, Abraira C, Moritz T, et al. Glucose control and vascular complications in veterans with type 2 diabetes. N Engl J Med 2009;360:129-39.

28. Bonds DE, Miller ME, Bergenstal RM, et al. The association between symptomatic, severe hypoglycaemia and mortality in type 2 diabetes: retrospective epidemiological analysis of the ACCORD study. BMJ 2010;340:b4909.

29. Zoungas S, Patel A, Chalmers J, et al. Severe hypoglycemia and risks of vascular events and death. N Engl J Med 2010;363:1410-18.

30. Riddle MC, Ambrosius WT, Brillon DJ, et al. Epidemiologic relationships between $\mathrm{A} 1 \mathrm{C}$ and all-cause mortality during a median 3.4-year follow-up of glycemic treatment in the ACCORD trial. Diabetes Care 2010;33:983-90.

31. Mannucci E, Monami M, Lamanna C, Gori F, Marchionni N. Prevention of cardiovascular disease through glycemic control in type 2 diabetes: a meta-analysis of randomized clinical trials. Nutr Metab Cardiovasc Dis 2009;19:604-12.

32. Ray KK, Seshasai SR, Wijesuriya S, et al. Effect of intensive control of glucose on cardiovascular outcomes and death in patients with diabetes mellitus: a meta-analysis of randomised controlled trials. Lancet 2009;373(9677):1765-72.

33. Boussageon R, Bejan-Angoulvant T, Saadatian-Elahi M, et al. Effect of intensive glucose lowering treatment on all cause mortality, cardiovascular death, and microvascular events in type 2 diabetes: meta-analysis of randomised controlled trials. BMJ 2011;343:d4169.

34. Klein R. Intensive treatment of hyperglycaemia: ACCORD. Lancet 2010;376(9739):391-2.

35. Handelsman Y, Mechanick JI, Blonde L, et al. American Association of Clinical Endocrinologists Medical Guidelines for Clinical Practice for developing a diabetes mellitus comprehensive care plan. Endocr Pract 2011;17(Suppl 2):1-53.

36. Rodbard HW, Jellinger PS, Davidson JA, et al. Statement by an American Association of Clinical Endocrinologists/ 
American College of Endocrinology consensus panel on type 2 diabetes mellitus: an algorithm for glycemic control. Endocr Pract 2009; 15:540-59.

37. Inzucchi SE, Bergenstal RM, Buse JB, et al. Management of hyperglycemia in type 2 diabetes: a patient-centered approach: position statement of the American Diabetes Association (ADA) and the European Association for the Study of Diabetes (EASD). Diabetes Care 2012;35:1364-79.

38. Cryer PE, Axelrod L, Grossman AB, et al. Evaluation and management of adult hypoglycemic disorders: an Endocrine Society Clinical Practice Guideline. J Clin Endocrinol Metab 2009;94:709-28.
39. National Kidney Foundation. K/DOQI clinical practice guidelines for chronic kidney disease: evaluation, classification and stratification. Am J Kidney Dis 2002;39(Suppl 1):S1-S266.

40. Chisholm JW, Goldfine AB, Dhalla AK, et al. Effect of ranolazine on $\mathrm{A} 1 \mathrm{C}$ and glucose levels in hyperglycemic patients with non-ST elevation acute coronary syndrome. Diabetes Care 2010;33:1163-8. 